Instituto Agronômico de Pernambuco - IPA, Av. General San Martin, 1371 CEP 50761-000, RECIFE, PE, Brasil ${ }^{2}$ Texas A \& M University - TAMU, COLLEGE STATION, TX, EUA

* autor correspondente $\bigotimes$ josimar.gurgel@ipa.br

\title{
Sistematização de dados de vegetação referente ao bioma caatinga para modelagem de bacias hidrológicas com SWAT
}

\author{
Systematization of vegetation data for the Caatinga biome for \\ SWAT hydrological basin modeling
}

Josimar Gurgel Fernandes ${ }^{*}$, Rita de Cássia Araújo Pereira Galindo ${ }^{1}$, Ana Luíza Du Bocage Neta ${ }^{1}$, Charles Allan Jones², Raghavan Srinivasan²

RESUMO: O SWAT é um modelo abrangente que requer uma diversidade de informações para ser executado. Usuários iniciantes podem se sentir um pouco impressionados com a variedade e o número de entradas quando começam a usar o modelo. No entanto, muitas das entradas são usadas para simular recursos especiais não comuns a todas as bacias hidrográficas. Esta nota científica fornece uma visão das entradas do modelo referente ao banco de dados de vegetação para modelagem de bacias hidrográficas. Isso ocorre em virtude das características particulares das plantas em cada ecossistema e bioma. Desse modo, a inserção do crescimento vegetal das culturas exploradas no Nordeste do Brasil, e também da vegetação nativa, possibilita gerar dados mais precisos e confiáveis para a modelagem, sendo as entradas organizadas por tópico. Este trabalho se concentra em ajudar o usuário a identificar entradas que devem ser definidas para seu conjunto de dados específico e apresentam as variáveis por arquivo, e discutem os métodos usados para medir ou calcular valores para os parâmetros de entrada, possibilitando assim quantificar a produção.

PALAVRAS-CHAVE: Crescimento vegetal, modelo, produção.

\begin{abstract}
SWAT is a comprehensive model that requires a wealth of information to be performed. Beginning users may be a little impressed by the variety and number of entries when they start using the template. However, many of the inputs are used to simulate special features that are not common to all watersheds. This scientific note provides an overview of model entries for the vegetation database for watershed grinding. This is due to the particular characteristics of the plants in each ecosystem. In this way, the insertion of plant growth of crops harvested in northeastern Brazil, as well as native vegetation, makes it possible to generate more accurate and reliable data for modeling. Being the entries organized by topics. This paper focuses on helping the user identify inputs that should be defined for their specific data set and present the variables by file and discuss the methods used to measure or calculate values for input parameters.
\end{abstract}

KEYWORDS: Plant growth, model, production.

\section{Referencial}

No modelo SWAT, as informações necessárias para simular o crescimento das plantas estão no o arquivo PLANT.DAT. Este arquivo de banco de dados é fornecido com o modelo. O banco de dados de crescimento de plantas distribuído com o SWAT inclui parâmetros para a maioria das espécies vegetais comuns. Se um usuário precisar modelar um uso da terra ou 
planta que não esteja contido no banco de dados, é possível determinar os parâmetros necessários da planta.

Cinco linhas são necessárias para armazenar os parâmetros de crescimento de plantas para uma cobertura de terra ou planta no banco de dados (plant.dat). $\mathrm{O}$ banco de dados de crescimento de plantas é um arquivo de formato livre. As variáveis podem ser colocadas em qualquer posição na linha, de acordo com o desejo do usuário. Os valores para as variáveis classificadas como inteiros não devem incluir um decimal, enquanto os valores das variáveis classificadas como reais devem conter um decimal. Um espaço em branco indica o final de um valor de entrada e o início do próximo valor, se houver outro na linha.

Segue-se uma breve descrição das variáveis de cobertura vegetal, junto com as informações necessárias para a modelação do crescimento da planta no SWAT. Elas são listadas na ordem em que aparecem dentro do arquivo.

\section{Nome da variável: ICNUM}

Definição: Código da cobertura / planta da terra.

As diferentes plantas listadas na base de dados de crescimento de plantas devem ter valores únicos para ICNUM. É o código numérico usado no arquivo de gerenciamento para identificar a cobertura de terra a ser modelada.

\section{Nome da variável: $\mathrm{CPNM}$}

Definição: Um código de quatro caracteres para representar a cobertura vegetal ou nome da planta.

Os códigos de quatro letras no crescimento de plantas e bases de dados urbanas são usados pelas interfaces SIG para vincular mapas de uso e cobertura da terra aos tipos de plantas SWAT. Este código é impresso para os arquivos de saída.

Ao adicionar uma nova espécie de planta ou categoria de cobertura da terra, o código de quatro letras para a nova planta deve ser exclusivo.

\section{Nome da variável: IDC}

Definição: Classificação da cobertura ou planta:

1 leguminosa anual de estação quente

2 leguminosa anual de estação fria

3 leguminosas perenes

4 temporada quente anual

5 estação fria anual

6 perenes

7 árvores

Processos modelados de forma diferente para os 7 grupos são:

1 leguminosa anual de estação quente

- simular a fixação de nitrogênio;

- a profundidade da raiz varia durante a estação de crescimento devido ao crescimento das raízes.

2 leguminosa anual de estação fria

- simular a fixação de nitrogênio;
- a profundidade da raiz varia durante a estação de crescimento devido ao crescimento das raízes;

- as coberturas de terras plantadas no outono ficarão adormecidas quando o comprimento do dia for menor que o limiar do comprimento do dia.

3 leguminosas perenes

- simular a fixação de nitrogênio;

- profundidade da raiz sempre igual ao máximo permitido para as espécies de plantas e solo;

- a planta fica adormecida quando o comprimento do dia é menor do que o limite de duração do dia.

4 temporada quente anual

- a profundidade da raiz varia durante a estação de crescimento devido ao crescimento das raízes.

5 estação fria anual

- a profundidade da raiz varia durante a estação de crescimento devido ao crescimento das raízes;

- as coberturas de terras plantadas no outono ficarão adormecidas quando o comprimento do dia for menor que o limiar do comprimento do dia.

6 perenes

- profundidade da raiz sempre igual ao máximo permitido para as espécies de plantas e solo;

- A planta fica dormente quando o dia é menor do que o limite do comprimento do dia.

7 árvores

- profundidade da raiz sempre igual ao máximo permitido para as espécies de plantas e solo;

- divide o novo crescimento entre folhas e espinhos (20\%) e crescimento lenhoso (80\%). Ao final de cada estação de crescimento, uma fração da biomassa é convertida em resíduo.

\section{Nome da variável: DESCRIPTION}

Definição: Cobertura total da terra ou nome da planta.

Esta descrição não é utilizada pelo modelo e está presente para auxiliar o utilizador na diferenciação entre espécies de plantas.

Nome da variável: BIO_E

Definição: Eficiência do uso de radiação ou razão biomassa-energia $\left(\left(\mathrm{kg}_{\mathrm{k}} \mathrm{ha}^{-1}\right) /\left(\mathrm{MJ} \cdot \mathrm{m}^{2}\right)\right)$.

A eficiência de uso de radiação (RUE) é a quantidade de biomassa seca produzida por unidade de radiação solar interceptada. A eficiência de uso de radiação é assumida como independente da fase de crescimento da planta. BIO E representa a taxa de crescimento potencial ou não estressada 
(incluindo raízes) por unidade de radiação fotossinteticamente ativa interceptada.

A determinação de RUE é comumente realizada, e uma revisão de literatura fornecerá aqueles que estabelecem experiências com numerosos exemplos. A seguinte síntese da metodologia utilizada para medir a RUE foi resumida a partir de Kiniry et al. (1998) e Kiniry et al. (1999).

Para calcular a RUE, a quantidade de radiação fotossinteticamente ativa (PAR) interceptada e a massa da biomassa acima do solo são medidas várias vezes ao longo da estação de crescimento de uma planta. A frequência das medições efectuadas irá variar, mas em geral 4 a 7 medições por estação de crescimento são consideradas adequadas. Tal como com determinações de área foliar, as medições devem ser realizadas em plantas não estressadas.

A radiação interceptada é medida com um medidor de luz. Estão disponíveis sensores de espectro inteiro e PAR e os cálculos de RUE serão realizados de forma diferente, dependendo do sensor utilizado. Uma breve discussão da diferença entre sensores de espectro inteiro e PAR e a diferença nos cálculos é dada em Kiniry (1999).

O uso de um sensor PAR nos estudos RUE é fortemente encorajado. Ao medir a radiação, três a cinco séries de medições são tomadas rapidamente para cada parcela vegetal. Um conjunto de medidas consiste em 10 medições acima da Folha, 10 abaixo e 10 mais acima. As medições de luz devem ser realizadas entre as 10:00 e as 14:00, hora local.

As medições acima e abaixo da copa das folhas são médias, e a fracção de PAR interceptada é calculada para o dia a partir dos dois valores. As estimativas diárias da fracção de PAR interceptada são determinadas interpolando linearmente os valores medidos.

A fracção de PAR interceptada é convertida numa quantidade de PAR interceptada utilizando valores diários de radiação solar total incidente medida com uma estação meteorológica padrão. Para converter a radiação incidente total em PAR total incidente, os valores diários de radiação solar são multiplicados pela percentagem de radiação total que tem um comprimento de onda entre 400 e $700 \mathrm{~mm}$. Esta porcentagem geralmente cai na faixa de 45 a $55 \%$, e é uma função da cobertura de nuvens. $50 \%$ é considerado como um valor padrão.

Uma vez que os valores de PAR interceptados diariamente são determinados, a quantidade total de PAR interceptada pela planta é calculada para cada data em que a biomassa foi colhida. Este valor é calculado pela soma dos valores diários interceptados de PAR a partir da data de emergência das plântulas até à data da colheita da biomassa.

Para determinar a produção de biomassa, a biomassa aérea é colhida de uma área conhecida de terra dentro da parcela. O material vegetal deve ser seco pelo menos 2 dias a $65^{\circ} \mathrm{C} \mathrm{e}$ depois pesado.

A RUE é determinada ajustando uma regressão linear para a biomassa acima do solo em função da PAR interceptada. A inclinação da linha é a RUE. A Figura 1 mostra as parcelas de biomassa acima do solo e somou a radiação fotossinteticamente ativa interceptada para o erva-de-gamão oriental. (Observe que as unidades para os valores RUE no gráfico, bem como os valores tipicamente relatados na literatura, são diferentes daqueles usados pelo SWAT. Para obter o valor usado no SWAT, multiplique por 10.)

Este parâmetro pode alterar muito a taxa de crescimento, a incidência de estresse durante a estação eo rendimento resultante. Este parâmetro deve ser um dos últimos a receber ajuste. Os ajustamentos devem basear-se nos resultados da investigação. Deve-se tomar cuidado para fazer ajustes com base apenas em dados sem estresse de seca, nutrientes ou temperatura.

\section{Nome da variável: HVSTI}

Definição: Índice de colheita para condições de crescimento ótimas.

O índice de colheita define a fração da biomassa aérea que é removida em uma operação de colheita. Este valor define a fracção de biomassa vegetal que está "perdida" do sistema e não está disponível para conversão em resíduo e subsequente decomposição. Para as culturas em que a parte colhida da planta está acima do solo, o índice de colheita é sempre uma fracção inferior a 1. Para as culturas em que a parte colhida está abaixo do solo, o índice de colheita pode ser superior a 1. Dois índices de colheita são fornecidos na base de dados: o índice de colheita para condições de crescimento ótimas (HVSTI) e o índice de colheita em condições de crescimento altamente estressadas (WSYF).

Para determinar o índice de colheita, a biomassa vegetal removida durante a operação de colheita é seca pelo menos dois dias a $65^{\circ} \mathrm{C}$ e pesada. A biomassa vegetal total acima do solo no campo também deve ser seca e pesada.

O índice de colheita é então calculado dividindo o peso da porção colhida da biomassa vegetal pelo peso da biomassa vegetal total acima do solo. As plantas terão de ser cultivadas em duas parcelas diferentes, em que as condições climáticas

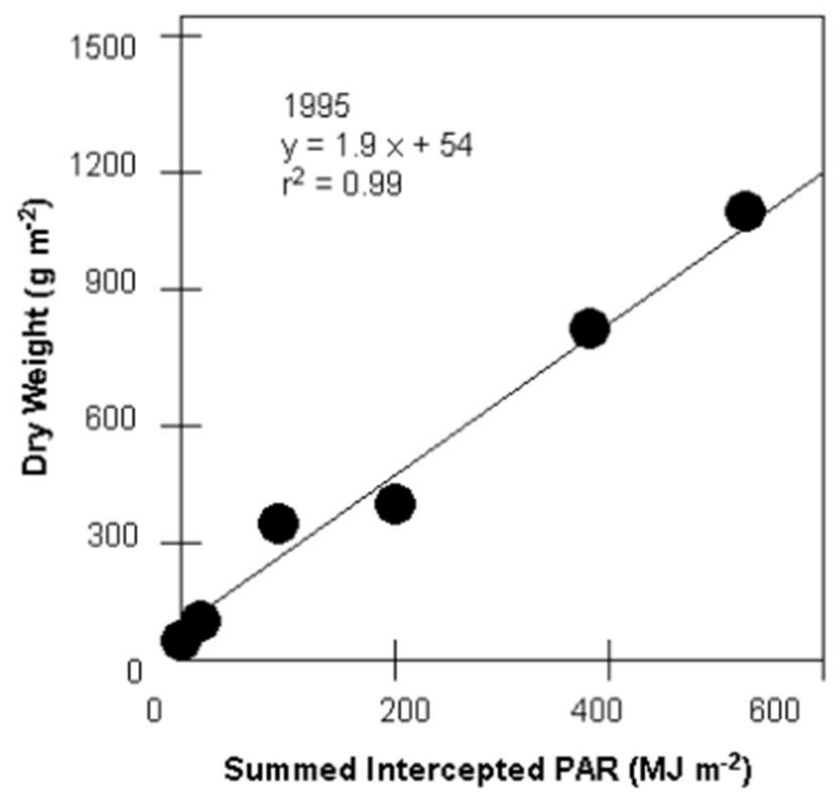

Figura 1. Biomassa acima do solo e radiação fotossinteticamente ativa somada interceptada para o gamagrass oriental (adaptado de Kiniry et al., 1999). 
ideais e condições de estresse são produzidas para obter valores para ambos os índices de colheita.

Nome da variável: BLAI

Definição: Índice de área foliar potencial máximo.

BLAI é um dos seis parâmetros utilizados para quantificar o desenvolvimento da área foliar de uma espécie vegetal durante a estação de crescimento.

A Figura 2 ilustra a relação dos parâmetros da base de dados com o desenvolvimento da área foliar modelado por SWAT.

Para identificar os parâmetros de desenvolvimento da área foliar, deve-se registrar o índice de área foliar e o número de unidades de calor acumuladas para as espécies de plantas ao longo da estação de crescimento, e depois traçar os resultados. Para melhores resultados, vários anos de dados de campo devem ser coletados. No mínimo, recomendam-se dados de dois anos. É importante que as plantas não sofram nenhum estresse de água ou nutrientes durante os anos em que os dados são coletados.

$\mathrm{O}$ índice de área foliar incorpora informações sobre a densidade da planta, de modo que as experiências de campo devem ser estabelecidas para reproduzir as densidades reais das plantas, ou o valor LAI máximo para a planta determinado a partir de experimentos de campo deve ser ajustado para refletir as densidades de plantas desejadas na simulação. Os valores máximos de LAI no banco de dados padrão correspondem às densidades de plantas associadas à agricultura de sequeiro.

O índice de área foliar é calculado dividindo a área de folha verde pela área de terra. Como a planta inteira deve ser colhida para determinar a área foliar, a experiência de campo precisa ser projetada para incluir plantas suficientes para acomodar todas as medições da área foliar feitas durante $o$ ano.

Embora a medição da área foliar possa ser laboriosa para amostras grandes, não há dificuldade intrínseca no processo. O método mais comum é obter um scanner eletrônico e alimentar as folhas verdes colhidas e hastes no scanner.

Os valores para BLAI na base de dados de crescimento de plantas baseiam-se na densidade média de plantas na agricultura de sequeiro (de sequeiro). O BLAI pode precisar ser ajustado para regiões propensas à seca onde as densidades de plantio são muito menores, ou irrigadas onde as densidades são muito maiores.

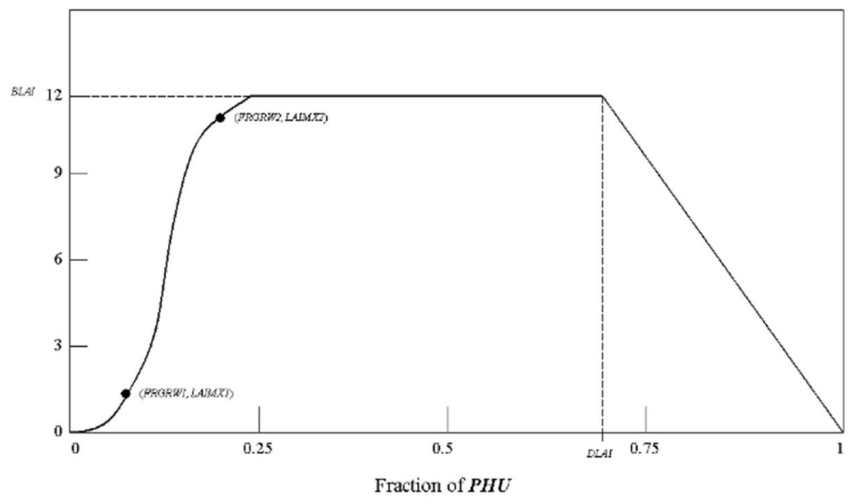

Figura 2. Índice de área foliar em função da fração da estação de crescimento de Alamo switchgrass (Arnold et al., 2012)

\section{Nome da variável: FRGRW1}

Definição: Fração da estação de crescimento da planta ou fração de unidades térmicas potenciais totais correspondente ao $1^{\circ}$ ponto na curva ótima de desenvolvimento da área foliar.

Consultar a Figura 2 e a explicação dada para o parâmetro BLAI para obter informações adicionais sobre este parâmetro e os métodos usados para medi-la.

Nome da variável: LAIMX1

Definição: Fração do índice de área foliar máximo correspondente ao $1^{\circ}$ ponto na curva de desenvolvimento da área foliar ideal.

Consultar a Figura 2 e a explicação dada para o parâmetro BLAI para obter informações adicionais sobre este parâmetro e os métodos usados para medi-la.

Nome da variável: FRGRW2

Definição: Fração da estação de crescimento da planta ou fração de unidades térmicas potenciais totais correspondente ao $2^{\circ}$ ponto da curva de desenvolvimento ótimo da área foliar.

Consultar a Figura 2 e a explicação dada para o parâmetro BLAI para obter informações adicionais sobre este parâmetro e os métodos usados para medi-la.

Nome da variável: LAIMX2

Definição: Fração do índice de área foliar máximo correspondente ao $2{ }^{\circ}$ ponto na curva de desenvolvimento da área foliar ideal.

Consultar a Figura 2 e a explicação dada para o parâmetro BLAI para obter informações adicionais sobre este parâmetro e os métodos usados para medi-la.

Nome da variável: DLAI

Definição: Fração da estação de crescimento quando a área foliar começa a declinar.

Consultar a Figura 2 e a explicação dada para o parâmetro BLAI para obter informações adicionais sobre este parâmetro e os métodos usados para medi-la.

Nome da variável: CHTMX

Definição: Altura máxima da copa (m).

A altura máxima do dossel é uma medida direta. A altura da copa das plantas não estressadas deve ser registrada em intervalos ao longo da estação de crescimento. O valor máximo registrado é usado no banco de dados.

\section{Nome da variável: RDMX}

Definição: Profundidade máxima da raiz (m).

Para determinar a profundidade máxima de enraizamento, as amostras de plantas precisam ser cultivadas em solos sem uma camada impermeável. Uma vez que as plantas atingiram a maturidade, os núcleos do solo são tomadao para toda a profundidade do solo. Cada incremento de 0,25 metro é lavado e o material vegetal vivo é coletado. Raízes vivas podem ser diferenciadas de raízes mortas porque que as raízes vivas são mais brancas e mais elásticas, e têm um córtex intacto. $\mathrm{O}$ incremento mais profundo do núcleo do solo no qual se encontram raízes vivas define a profundidade máxima de enraizamento.

Nome da variável: T_OPT

Definição: Temperatura ideal para o crescimento das plantas $\left({ }^{\circ} \mathrm{C}\right)$.

As temperaturas ótimas e baixas são muito estáveis para cultivares dentro de uma espécie. A temperatura ideal para 
o crescimento da planta é difícil de medir diretamente. Observando a Figura 3, pode ser tentado selecionar a temperatura correspondente ao pico do gráfico como a temperatura ótima. Isso não seria correto.

O pico da parcela define a temperatura ideal para o desenvolvimento foliar, não para o crescimento da planta. Se uma temperatura ótima não pode ser obtida através de uma revisão da literatura, use a temperatura ideal listada para uma planta já no banco de dados com hábitos de crescimento semelhantes.

A revisão das temperaturas para muitas plantas diferentes forneceu valores genéricos para temperaturas de base e ótimas em função da estação de crescimento. Em situações nas quais as informações de temperatura não estão disponíveis, esses valores podem ser usados. Para plantas de estação quente, a temperatura de base genérica é $\sim 8^{\circ} \mathrm{C}$, e a temperatura ótima genérica é $\sim 25^{\circ} \mathrm{C}$. Para plantas de estação fria, a temperatura de base genérica é $\sim 0^{\circ} \mathrm{C}$ e a temperatura ótima genérica é $\sim 13^{\circ} \mathrm{C}$.

Nome da variável: $T$ BASE

Definição: Temperatura mínima (base) para o crescimento das plantas $\left({ }^{\circ} \mathrm{C}\right)$.

SWAT usa a temperatura de base para calcular o número de unidades de calor acumuladas todos os dias. A temperatura mínima ou base para o crescimento da planta varia com a fase de crescimento da planta. No entanto, esta variação é ignorada pelo modelo SWAT, que usa a mesma temperatura base ao longo da estação de crescimento.

A temperatura de base é medida pelo crescimento de plantas em câmaras de crescimento a várias temperaturas diferentes. A taxa de aparência da ponta da folha em função da temperatura é traçada. A extrapolação da linha à taxa de aparência da ponta da folha de 0,0 folhas.dia ${ }^{-1}$ dá a base ou temperatura mínima para o crescimento da planta. A Figura 3 apresenta os dados para o milho. (Note que a linha intersecta o eixo x a $8{ }^{\circ} \mathrm{C}$.)

Nome da variável: CNYLD

Definição: Fração normal de azoto em rendimento ( $\mathrm{kg} \mathrm{N} / \mathrm{kg}$ de rendimento).

Além da quantidade de biomassa vegetal removida no rendimento, SWAT precisa saber a quantidade de nitrogênio e

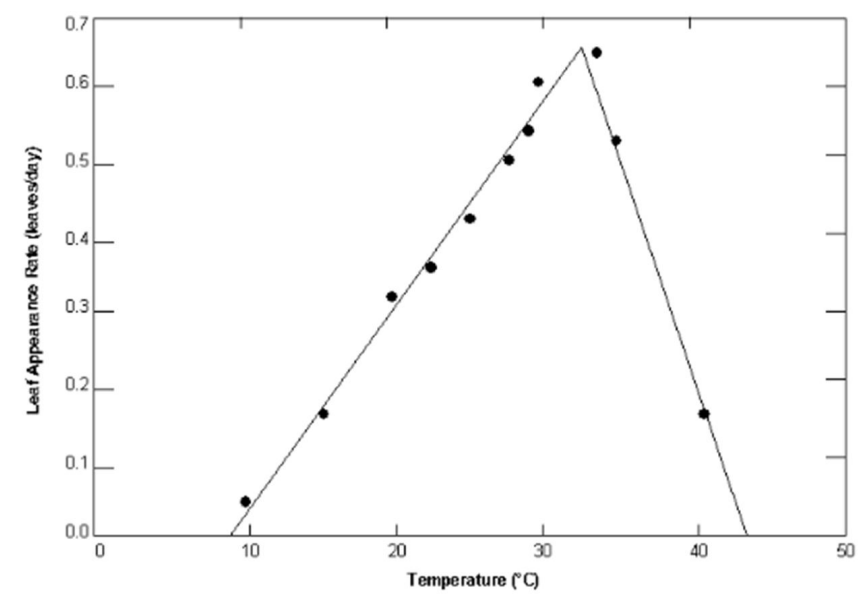

Figura 3. Taxa de aparência da ponta da folha em função da temperatura do milho (Kiniry et al., 1991 apud Arnold et al., 2012). fósforo removidos no rendimento. A parte colhida da biomassa vegetal é enviada para um laboratório de testes para determinar a fração de nitrogênio e fósforo na biomassa. Este valor é estimado numa base de peso seco.

Nome da variável: CPYLD

Definição: Fração normal de fósforo em rendimento ( $\mathrm{kg} \mathrm{P} / \mathrm{kg}$ de rendimento).

Além da quantidade de biomassa vegetal removida no rendimento, SWAT precisa saber a quantidade de nitrogênio e fósforo removida no rendimento. A parte colhida da biomassa vegetal é enviada para um laboratório de testes para determinar a fração de nitrogênio e fósforo na biomassa. Este valor é estimado numa base de peso seco.

Nome da variável: PLTNFR (1)

Definição: Parâmetro de captação de nitrogênio \# 1: fração normal de nitrogênio na biomassa vegetal na emergência ( $\mathrm{kg} \mathrm{N} / \mathrm{kg}$ de biomassa).

Para calcular a demanda de nutrientes de plantas ao longo do ciclo de crescimento de uma planta, a SWAT precisa conhecer a fração de nutriente na biomassa total da planta (em base de peso seco) em diferentes estágios de crescimento da cultura. Seis variáveis na base de dados da planta fornecem esta informação: PLTNFR (1), PLTNFR (2), PLTNFR (3), PLTPFR (1), PLTPFR (2) e PLTPFR (3). As amostras de plantas são analisadas quanto ao teor de nitrogênio e fósforo três vezes durante a estação de crescimento: logo após a emergência, perto da metade da estação e na maturidade. As amostras de plantas podem ser enviadas para laboratórios de teste para obter a fração de nitrogênio e fósforo na biomassa.

Idealmente, as amostras de plantas testadas quanto ao teor de nutrientes devem incluir as raízes, bem como a biomassa acima do solo. Diferenças na partição de nutrientes para raízes e brotos podem causar conclusões errôneas quando se compara a produtividade entre espécies, se apenas a biomassa acima do solo for medida.

Nome da variável: PLTPFR (2)

Definição: Parâmetro \# 2 de captação de fósforo: fração normal de fósforo na biomassa vegetal a $50 \%$ de maturidade ( $\mathrm{kg} \mathrm{P} / \mathrm{kg}$ de biomassa).

Ler a explicação para o parâmetro PLTNFR (1) para obter informações adicionais sobre este parâmetro e os métodos utilizados para medi-lo.

Nome da variável: PLTPFR (3)

Definição: Parâmetro de captação de fósforo \# 3: fração normal de fósforo na biomassa vegetal na maturidade ( $\mathrm{kg} \mathrm{P} / \mathrm{kg}$ de biomassa).

Ler a explicação para o parâmetro PLTNFR (1) para obter informações adicionais sobre este parâmetro e os métodos utilizados para medi-lo.

Nome da variável: WSYF

Definição: Limite inferior do índice de colheita $\left(\left(\mathrm{kg} \cdot \mathrm{ha}^{-1}\right) /\left(\mathrm{kg} \cdot \mathrm{ha}^{-1}\right)\right)$.

$\mathrm{O}$ valor entre 0,0 e HVSTI, que representa o menor índice de colheita esperado devido ao estresse hídrico.

Ler a explicação para o parâmetro HVSTI para obter informações adicionais sobre este parâmetro e os métodos usados para medi-lo. 
Nome da variável: USLE $\mathrm{C}$

Definição: Valor mínimo do fator USLE C para a erosão hídrica aplicável à cobertura / instalação vegetal.

$\mathrm{O}$ fator $\mathrm{C}$ mínimo pode ser estimado a partir de um fator $\mathrm{C}$ anual médio conhecido usando a seguinte equação (ARNOLD; WILLIAMS, 1995):

$C_{U S L E, m n}=1.463 \ln \left(C_{U S L E, a a}\right)+0,1034$

Onde $\mathrm{C}_{U S L E, m n}$ é o fator $\mathrm{C}$ mínimo para a cobertura da terra e $\mathrm{C}_{U S L E, a a}$ é o fator $\mathrm{C}$ anual médio para a cobertura da terra.

Nome da variável: GSI

Definição: Máxima condutância estomática em altas radiações solares e baixo déficit de pressão de vapor $\left(\mathrm{m} \mathrm{s}^{-1}\right)$.

A condutância estomática do vapor de água é utilizada nos cálculos de Penman-Monteith da evapotranspiração máxima da planta. A base de dados da planta contém três variáveis referentes à condutância estomática que são necessárias apenas se as equações de Penman-Monteith forem escolhidas para modelar a evapotranspiração: condutância estomática máxima (GSI) e duas variáveis que definem o impacto do déficit de pressão de vapor na condutância estomática (FRGMAX, VPDFR).

Körner et al. (1979) definem a máxima condutância difusiva foliar como o maior valor de condutância observado em folhas totalmente desenvolvidas de plantas bem irrigadas sob condições climáticas ótimas, concentrações naturais de $\mathrm{CO}_{2}$ ao ar livre e suprimento de nutrientes suficiente. A condutância difusiva da folha de vapor de água não pode ser medida diretamente, mas pode ser calculada a partir de medidas de transpiração em condições climáticas conhecidas. Diversos métodos são utilizados para determinar a condutância difusiva: medidas de transpiração em cuvetes de fotossíntese, medições de balanço de energia ou experimentos de pesagem, poros de difusão ventilada e poros não ventilados.

Para obter valores máximos de condutância foliar, a condutância foliar é determinada entre o nascer e o fim da manhã, até se observar um claro declínio ou nenhum aumento adicional. Dependendo da fenologia, as medições são tomadas em pelo menos três dias brilhantes no final da primavera e verão, de preferência logo após um período chuvoso. As médias de condutância foliar máxima de 5 a 10 amostras por dia são médias, obtendo-se a condutância máxima difusiva para a espécie. Devido à variação da localização dos estômatos nas folhas de plantas para diferentes espécies de plantas, os valores de condutância devem ser calculados para a área total da superfície foliar.

\section{Nome da variável: VPDFR}

Definição: Déficit de pressão de vapor $(\mathrm{kPa})$ correspondente ao segundo ponto da curva de condutância estomática.

O primeiro ponto da curva de condutância estomática é composto por um déficit de pressão de vapor de $1 \mathrm{kPa}$ e a fração de condutância estomática máxima igual a 1,00. Tal como com a eficiência do uso da radiação, a condutância estomática é sensível ao déficit de pressão de vapor. Stockle et al. (1992) compilaram uma pequena lista de resposta de condutância estomática ao déficit de pressão de vapor para algumas espécies de plantas. Devido à escassez de dados, os valores padrão para o segundo ponto da condutância estomática versus curva de déficit de pressão de vapor são usados para todas as espécies de plantas no banco de dados. A fração de condutância estomática máxima (FRGMAX) é ajustada para 0,75 , e o déficit de pressão de vapor correspondente à fração dada por FRGMAX (VPDFR) é ajustado para $4,00 \mathrm{kPa}$. Se o usuário tiver dados reais, eles devem usar esses valores, caso contrário, os valores padrão são adequados.

\section{Nome da variável: FRGMAX}

Definição: Fração da condutância estomática máxima correspondente ao segundo ponto da curva de condutância estomática. O primeiro ponto da curva de condutância estomática é composto por um déficit de pressão de vapor de $1 \mathrm{kPa}$ e a fração de condutância estomática máxima igual a 1,00. Ler a explicação para o parâmetro VPDFR para obter informações adicionais sobre este parâmetro e os métodos usados para medi-lo.

Nome da variável: WAVP

Definição: Taxa de declínio na eficiência de uso de radiação por unidade de aumento no déficit de pressão de vapor.

Stockle e Kiniry (1990) observaram pela primeira vez uma relação entre a RUE e o déficit de pressão de vapor, e foram capazes de explicar uma grande parcela da variabilidade intraespécies nos valores de RUE para sorgo e milho ao traçar os valores de RUE em função dos valores médios de déficit de pressão de vapor diário. Desde este primeiro artigo, uma série de outros estudos têm sido realizados que apoiam a dependência da RUE sobre o déficit de pressão de vapor. No entanto, ainda há algum debate na comunidade científica sobre a validade dessa relação. Se o usuário não desejar simular uma alteração na RUE com déficit de pressão de vapor, a variável WAVP pode ser ajustada para 0,0 para a planta.

Para definir o impacto do déficit de pressão de vapor na RUE, valores de déficit de pressão de vapor devem ser registrados durante as estações de crescimento em que estão sendo feitas determinações de RUE. É importante que as plantas não sejam expostas a nenhum outro estresse de déficit de pressão de vapor, isto é, o crescimento de plantas não deve ser limitado pela falta de água e nutrientes no solo.

Os déficits de pressão de vapor podem ser calculados a partir da umidade relativa ou das temperaturas máximas e mínimas diárias usando a técnica de Diaz e Campbell (1988), como descrito por Stockle e Kiniry (1990). A variação da RUE com o déficit de pressão de vapor é determinada ajustando uma regressão linear para a RUE em função do déficit de pressão de vapor. Figura 4 mostra um gráfico de RUE em função do déficit de pressão de vapor para sorgo de grãos.

$\mathrm{Na}$ Figura 4, a taxa de declínio na eficiência de uso de radiação por unidade de aumento no déficit de pressão de vapor, $\Delta r u e_{d c l}$, para o sorgo é $8,4 \times 10^{-1} \mathrm{~g} \cdot \mathrm{MJ}^{-1} \cdot \mathrm{kPa}^{-1}$. Quando RUE é ajustado para o déficit de pressão de vapor, o modelo assume que o valor RUE relatado para BIO_E é a eficiência de uso de radiação em um déficit de pressão de vapor de $1 \mathrm{kPa}$.

$O$ valor de WAVP varia entre as espécies, mas um valor de 6 a 8 é sugerido como uma aproximação para a maioria das plantas.

Nome da variável: $\mathrm{CO}_{2} \mathrm{HI}$

Definição: Concentração atmosférica elevada de $\mathrm{CO}_{2}$ ( $\mu \mathrm{L} \mathrm{CO}_{2} / \mathrm{L}$ de ar) correspondente ao $2^{\circ}$ ponto da curva de eficiência de utilização da radiação. 


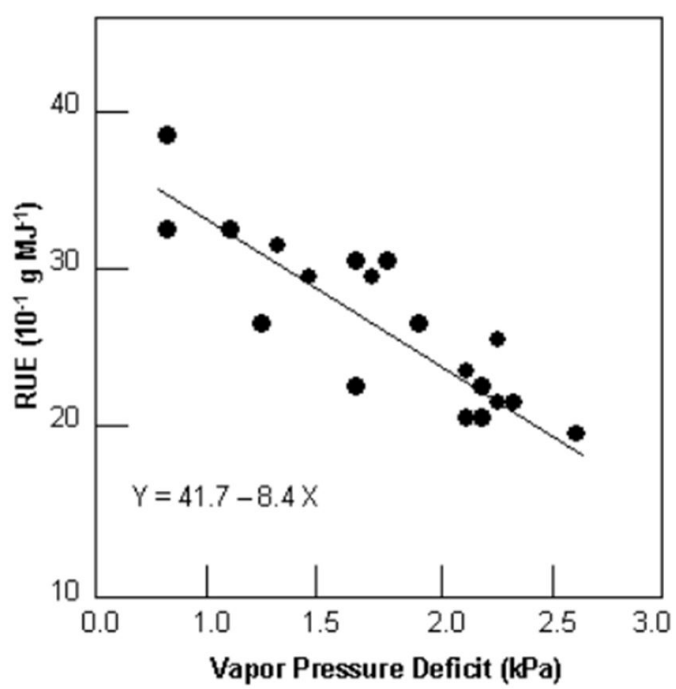

Figura 4. Resposta da eficiência de uso de radiação para significar déficit de pressão de vapor diário para sorgo de grãos (adaptado de Kiniry, 1999).

O primeiro ponto da curva de eficiência de uso de radiação é composto pela concentração de $\mathrm{CO}_{2}$ ambiente, $330 \mu \mathrm{L}$ de $\mathrm{CO}_{2} \cdot \mathrm{L}^{-1}$ de ar e a relação biomassa-energia relatada para BIO_E.

Para avaliar o impacto das alterações climáticas na produtividade agrícola, a SWAT incorpora equações que ajustam a RUE para concentrações elevadas de $\mathrm{CO}_{2}$ atmosférico. Os valores devem ser inseridos para $\mathrm{CO}_{2} \mathrm{HI}$ e BIOEHI no banco de dados da planta, quer o usuário planeje ou não simular mudanças climáticas.

Para simulações nas quais os níveis elevados de $\mathrm{CO}_{2}$ não são modelados, $\mathrm{CO}_{2} \mathrm{HI}$ deve ser ajustado para algum número maior que 330 ppmv, e BIOEHI deve ser definido para algum número maior do que BIO_E.

Para obter valores de eficiência de uso de radiação em níveis elevados de $\mathrm{CO}_{2}$ para espécies de plantas não atualmente na base de dados, as plantas devem ser estabelecidas em câmaras de crescimento instaladas no campo ou laboratório onde os níveis de $\mathrm{CO}_{2}$ podem ser controlados. Os valores de RUE são determinados usando a mesma metodologia descrita na explicação de BIO_E.

Nome da variável: BIOEHI

Definição: Relação biomassa-energia correspondente ao $2^{\circ}$ ponto da curva de eficiência de uso de radiação.

Ler a explicação para o parâmetro $\mathrm{CO}_{2} \mathrm{HI}$ e BIO_E para obter informações adicionais sobre este parâmetro e os métodos usados para medi-lo.

Nome da variável: RSDCO_PL

Definição: Coeficiente de decomposição de resíduos de plantas.

O coeficiente de decomposição de resíduos de plantas é a fração de resíduo que se decomporá em um dia assumindo a umidade, temperatura, relação $\mathrm{C}: \mathrm{N}$ e relação $\mathrm{C}$ : $\mathrm{P}$ ótima.

Esta variável estava originalmente no arquivo de entrada da bacia (.bsn), mas foi adicionada à base de dados de culturas para que os usuários pudessem variar a decomposição por espécies de plantas. Um valor padrão de 0,05 é usado para todas as espécies de plantas no banco de dados.

Nome da variável: ALAI_MIN
Definição: Índice mínimo de área foliar para plantas durante o período de dormência $\left(\mathrm{m}^{2} \cdot \mathrm{m}^{2}\right)$.

Esta variável refere-se apenas a plantas perenes e árvores. (O valor nunca é usado para outros tipos de plantas.) Nas versões do SWAT anteriores ao SWAT 2012, o índice mínimo de área foliar para as plantas durante o período de latência foi sempre ajustado para 0,75 . Como esse valor não era ideal para todas as plantas (árvores em particular), os usuários agora podem variar o LAI mínimo para a dormência.

Consulte a explicação dada para o parâmetro BLAI para obter informações adicionais sobre este parâmetro e os métodos usados para medi-lo.

Nome da variável: BIO_LEAF

Definição: Fração de biomassa de árvore acumulada a cada ano que é convertida em resíduo durante a dormência.

Esta variável refere-se apenas às árvores. (O valor nunca é usado para outros tipos de plantas.) BIO_LEAF regula a quantidade de biomassa que cai da árvore e é convertido em resíduo quando a planta fica inativa no inverno. Nas versões do SWAT anteriores ao SWAT 2012, a fração de biomassa convertida em resíduo no início da dormência foi sempre definida como 0,30 . Necessário se a cobertura da terra for classificada como uma árvore (ver IDC).

Nome da variável: MAT_YRS

Definição: Número de anos necessários para que as espécies de árvores alcancem pleno desenvolvimento (anos).

Esta variável refere-se apenas às árvores. (O valor nunca é usado para outros tipos de plantas.) Obrigatório se a cobertura da terra for classificada como uma árvore (ver IDC).

Nome da variável: BMX_TREES

Definição: Biomassa máxima para uma floresta (toneladas métricas.ha $\left.{ }^{-1}\right)$.

Esta variável refere-se apenas às árvores. (O valor nunca é usado para outros tipos de plantas.)

A biomassa máxima para um bosque maduro geralmente fica na faixa de 30-50 toneladas métricas.ha ${ }^{-1}$. Obrigatório se a cobertura da terra for classificada como uma árvore (ver IDC).

Nome da variável: BMDIEOFF

Definição: Fração de morte de biomassa.

Este coeficiente é a fração da biomassa acima do solo que morre na dormência. Valor padrão $=0,10$.

Nome da variável: RSR1C

Definição: Proporção inicial de brotamento radicular no início da estação de crescimento. Padrão = 0,40.

Nome da variável: RSR2C

Definição: Proporção inicial de brotamento radicular no final da época de crescimento. Padrão $=0,20$.

Nome da variável: EXT_COEF

Definição: Coeficiente de extinção da luz.

Este coeficiente é usado para calcular a quantidade de radiação fotossinteticamente ativa interceptada. Nas versões do SWAT anteriores ao SWAT 2012, o coeficiente de extinção da luz foi sempre definido como 0,65 .

As diferenças na estrutura do dossel de uma espécie são descritas pelo número de folhas presentes (índice de área foliar) e pela orientação foliar. A orientação das folhas tem um impacto significativo na interceptação de luz e, 


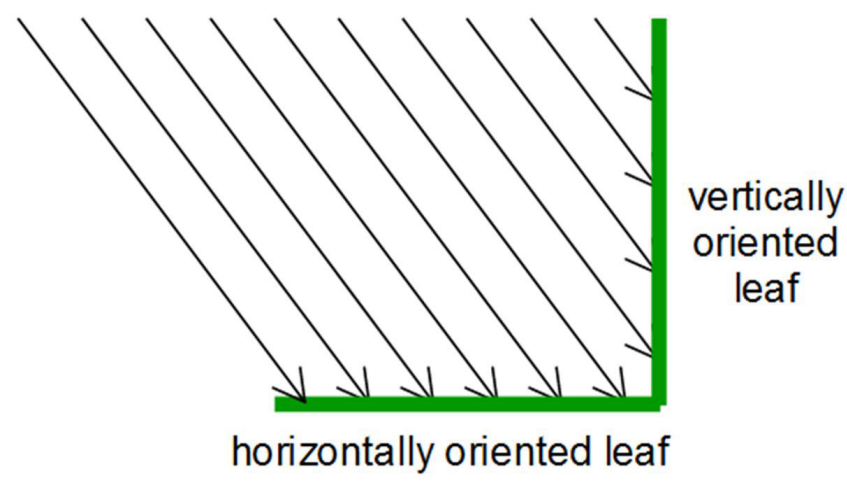

Figura 5. Interceptação da intensidade da luz em função da orientação foliar. A folha orientada verticalmente intercepta 4 unidades de luz, enquanto uma folha horizontalmente orientada do mesmo comprimento intercepta 6 unidades de luz. (Arnold et al., 2012).

consequentemente, na eficiência do uso da radiação. Tipos de folhas mais eretas espalham a luz de entrada sobre uma maior área foliar, diminuindo a intensidade média da luz interceptada por folhas individuais (Figura 5). Uma redução na intercepção da intensidade luminosa por uma folha individual favorece uma conversão mais completa da energia luminosa total interceptada pelo dossel em biomassa.

Utilizando o valor do coeficiente de extinção de luz $(\mathrm{k} \ell)$ na fórmula de Beer-Lambert para quantificar a eficiência de interceptação de luz por unidade de índice de área foliar, os tipos de folhas mais eretas têm um $\mathrm{k} \ell$ menor.

Para calcular o coeficiente de extinção da luz, mede-se a quantidade de radiação fotossinteticamente ativa (PAR) interceptada e a massa de biomassa aérea (LAI) várias vezes ao longo da estação de crescimento de uma planta usando a metodologia descrita nas seções anteriores. O coeficiente de extinção da luz é então calculado utilizando a equação de Beer-Lambert:

$$
\frac{T P A R}{P A R}=\left(1-\exp \left(-k_{\ell} \cdot L A I\right)\right) o u k_{\ell}=-\ln \left(\frac{T P A R}{P A R}\right) \cdot \frac{1}{L A I}
$$

Onde TPAR é a radiação fotossinteticamente ativa transmitida, e PAR é a radiação fotossinteticamente ativa que entra.

O banco de dados de vegetação referente ao bioma caatinga para modelagem com o SWAT está disponível aos usuários no arquivo suplementar a esse artigo.

\section{Referências}

ARNOLD. J.G.; KINIRY, J.R.; SRINIVASAN, R.; WILLIAMS, J.R.; HANEY, E.B.; NEITSCH, S.L. SWAT 2012 Input/Output Documentation. Texas: Texas Water Resources Institute, 2012. Available from: <http://hdl.handle.net/1969.1/149194>. Access in: 03 October 2018

ARNOLD, J. G.; WILLIAMS, J. R. SWRRB - A watershed scale model for soil and water resources management. In: SINGH, V. P. (Ed.). Computer models of watershed hydrology. Highlands Ranch: Water Resources Publications, 1995. p. 847-908.

DIAZ, R. A.; CAMPBELL, G. S. Assessment of vapor density deficit from available air temperature information. Anaheim: ASA Annual Meetings Agronomy Abstracts, 1988. 16 p.

KINIRY, J. R. Response to questions raised by Sinclair and Muchow. Field Crops Research, Amsterdam, v. 62, p. 245-247, 1999.

KINIRY, J. R. et al. Radiation-use efficiency response to vapor pressure deficit for maize and sorghum. Field Crops Research, Amsterdam, v. 56, p. 265-270, 1998.

KINIRY, J. R.; TISCHLER, C. R.; VAN ESBROECK, G. A. Radiation use efficiency and leaf $\mathrm{CO} 2$ exchange for diverse $\mathrm{C} 4$ grasses. Biomass and Bioenergy, Oxford, v. 17, p. 95-112, 1999.

KÖRNER, C.; SCHEEL, J. A.; BAUER, H. Maximum leaf diffusive conductance in vascular plants. Photosynthetica, Germany, v. 13 , p. $45-82,1979$.

STOCKLE, C. O.; KINIRY, J. R. Variability in crop radiation-use efficiency associated with vapor pressure deficit. Field Crops Research, Amsterdam, v. 25, p. 171-181, 1990.

STOCKLE, C. O. et al. A method for estimating the direct and climatic effects of rising atmospheric carbon dioxide on growth and yield of crops: Part 1-Modification of the EPIC model for climate change analysis. Agricultural Systems, Elsevier, v. 38, p. 225-238, 1992.

Recebido: 03 out. 2018 Aprovado: 06 nov. 2019 


\section{Material Suplementar}

Material suplementar acompanha este artigo.

Tabela S1. Estruturação do bando de dados (plant.dat) adaptado ao bioma caatinga.

Este material está disponível como parte do artigo online publicado na página https://pap.emnuvens.com.br/pap/. 